\title{
Implementation of Mobile Target Positioning Technology Integrating SINS with WSN Measurements
}

\author{
Chengming Luo, Wei Li, Hai Yang, Baohua Ying, and Gaifang Xin \\ School of Mechatronic Engineering, China University of Mining and Technology, Xuzhou, Jiangsu 221116, China \\ Correspondence should be addressed to Wei Li; cmeecumt512@yahoo.com
}

Received 19 April 2014; Revised 12 August 2014; Accepted 20 August 2014; Published 1 September 2014

Academic Editor: Andrea Cusano

Copyright (c) 2014 Chengming Luo et al. This is an open access article distributed under the Creative Commons Attribution License, which permits unrestricted use, distribution, and reproduction in any medium, provided the original work is properly cited.

\begin{abstract}
Accurate and robust positioning technology is expected to promote management level and service efficiency in various industrial applications. The strap-down inertial navigation system (SINS) approach has short-term accurate positioning performance, but the SINS is known for its accumulative error over time. Meanwhile, wireless sensor networks (WSN) approach can keep the mobile target on effective tracking after a long time monitoring, but the WSN may have large positioning error in certain areas. In order to make the positioning method allow profit from their advantages, this paper proposes a positioning technology using SINS approach in conjunction with WSN approach. The measurement parameters by SINS and WSN approaches are used. Then the SINS, anchor nodes, mobile tags, XBee, and computer are applied to design the positioning system. The estimation results indicate that the proposed method can make up for the shortcomings by pure SINS or WSN method and can be available for some accurate and robust applications.
\end{abstract}

\section{Introduction}

1.1. Background. Positioning estimation for mobile target is considered a key technology that can greatly help in improving the management level and service efficiency [1]. In many engineering and science applications, the sensors need to acquire their positions to report where a certain event takes place. The positioning system is designed with very stringent requirements for the accurate and robust positioning [2]. For instance, aircrafts dynamically adjust their trajectory paths using the estimated positions; positioning service of public transports can be provided to inform the passengers when the buses arrive at the station; miners in the underground can rescue their lives by specifying the locations when mine accidents take place; indoor positioning systems are designed to track inmates, patient, and fire fighters. Various practical approaches [3], such as global positioning systems (GPS), strap-down inertial navigation system (SINS), wireless sensor networks (WSN), vision odometer, and laser have been used to research.

1.2. Related Works. The SINS approach has been an autonomous method in positioning services. Most SINS methods employ inertial sensing elements of three-axis accelerometer (TAA) and three-axis gyroscope (TAG) to measure the acceleration and angular velocity values [4]. Combining the initial inertia values, mobile target has a short term accurate positioning estimation through calculating quadratic integration based on Newtonian mechanics theorem. Lü et al. [5] developed a positioning method for mine personnel using SINS. After the hardware and software design are accomplished, the positioning system can resolve the pose, velocity, and location. Hoflinger et al. [6] designed the SINS method in combination with CC430 microcontroller. Then the INS placed on a shoe was used to evaluate pedestrian tracking indoors. Kalman filter (KF) method was adopted to optimally estimate and compensate for inertial errors, which can improve the SINS positioning performance [7]. In order to improve the performance of present inertial sensors, innovative lasers and fiber optic gyro technologies are all of interest for inclusion in SINS.

Scholars focus on position and pose estimation by SINS approach. However, the accelerometers of SINS are known for their drifts over time when suffering from multinoises [8]. Owing to the fact that the estimation position purely by SINS method has accumulative error after a long time, 
the SINS system needs to benefit from the aid of other positioning systems. In order to eliminate the accumulative error caused by SINS, GPS method can help correct the increasing position errors of pure SINS approach. The SINS/GPS integration applications are widely used to track the aircrafts or vehicles in open environments [9]. However in enclosed environments, such as indoors, urban, and underground mines where GPS is unavailable, the GPS even cannot provide the position and velocity of mobile targets due to heavy satellite signal blockage. Hence, how to weaken the SINS accumulative error over time is a meaningful research with the aid of other sensors after keeping a long-term tracking.

Compared with SINS method, WSN method can be realized using the distance measurements among anchor nodes and mobile tags extracted from the wireless signals. WSN has the ability to sense, process, and communicate and can form a network map and estimates position by using the wireless signals of received signal strength indicator (RSSI), time of arrival (TOA), time differences of arrival (TDOA), and angle of arrival (AOA) measurements [10]. Various methods have been extensively utilized to estimate the position of mobile target. Mazuelas et al. [11] only proposed a novel positioning algorithm using RSSI approach, which can make propagation models fit the propagation environments best. Chan and Ho [12] proposed a simple and efficient method to estimate the position of mobile tags using TDOA approach. Compared with the existing methods, the accuracy of proposed positioning algorithm by Chan method can attain the Cramer-Rao lower bound (CRLB) accuracy. The CRLB method is regarded as a useful tool and can help us judge the designed positioning algorithm. Jiang et al. [13] addressed distributed positioning method using the RSSI approach as the most suitable measurement technique. The estimation results had high accuracy through establishing the geometric relationship between anchor nodes and mobile tags. Considering the industrial environments, an AOA approach of ultrawide band (UWB) had been investigated under multipath propagation environments [14].

WSN positioning is a kind of distributed positioning method which has no accumulative error after a long-term racking. However, the position accuracy of WSN method can be affected by the environment conditions. Line of sight (LOS) may make the WSN have accurate positioning results, while the nonline of sight (NLOS) may lead to large positioning errors due to signal outage and multipath propagation. The existing literatures prove that more effective information can provide accurate and robust estimations that can eliminate the shortcomings of noise measurements. The SINS method has short term accurate positioning, but the SINS is known for its accumulative error. Meanwhile, the WSN method can keep the mobile target on effective tracking, but the WSN may suffer from large positioning errors in certain areas. Note that the SINS and WSN positioning methods are available, but they have their own disadvantages. Hence, how to make full use of the advantages of SINS and WSN approaches is vital to improve the positioning performance. In order to combine two approaches advantages, the SINS/WSN integration needs to be studied.
As an effort to overcome the limitations of single sensor, intelligent filtering methods, such as Kalman filter (KF), extended Kalman filter (EKF), and unscented Kalman filter (UKF), are required to improve the positioning performance. WSN integrated with SINS method makes tracking performance present optimal estimation results [15]. Zhu et al. [16] expanded the SINS/WSN positioning method in coal mine environment, which can provide the accurate velocity and position of mobile vehicles. Ascher et al. [17] presented an innovative monitoring method that applied the SINS/UWB technology to locate pedestrian indoors. Hol et al. [18] designed 6 degrees of freedom (DOF) tracking system through combining the SINS with UWB approach. The experimental results show that the proposed method always has great tracking performance. The EKF method was utilized based on SINS and UWB approaches. Then SINS/UWB integration method can provide dynamic test scenario experimental results [19]. Zhang et al. [20] utilized the EKF method to make radio frequency identification (RFID) and SINS approaches efficient. Then SINS/RFID integration method can produce a three-dimensional positioning estimation.

1.3. Motivation of Our Work. Accurate and robust positioning technology is required in various engineering and science fields. The SINS or WSN approach can solve the mobile target position alone. However, the SINS method is known for its accumulative error and the WSN method may have large positioning errors. Hence in order to enhance two approaches advantages, the tightly coupled SINS/WSN tightly coupled needs to be intensively studied. Based on the above mentioned analysis, the KF or EKF has been applied to the integrated positioning system. This paper proposed a collaborative positioning algorithm combining information from the SINS and WSN approaches. The SINS approach measures three-axis accelerations and three-axis angular rates of mobile target, while the WSN detects the distance values among anchor nodes and mobile tag. The measured parameters of SINS and WSN approaches are used to establish solution equations based on UKF method. The SINS/WSN integration can avoid the limitations brought by single measurement. The actual positioning system prototype is designed for evaluating the proposed positioning method.

1.4. Organization of This Paper. The second section gives the block diagram of the SINS integrated with WSN method. The third section introduces the adopted sensors and instruments and implements pure SINS and WSN positioning approach. The next section implements the actual experiments and evaluates the proposed SINS/WSN positioning. Section 5 concludes this paper.

\section{System Architecture}

Figure 1 shows the block diagram of SINS and WSN methods. The mobile target moves around with the SINS and mobile tag, while the anchor nodes are deployed in the monitoring area. SINS method provides angle, velocity, and location information; WSN method provides single position 


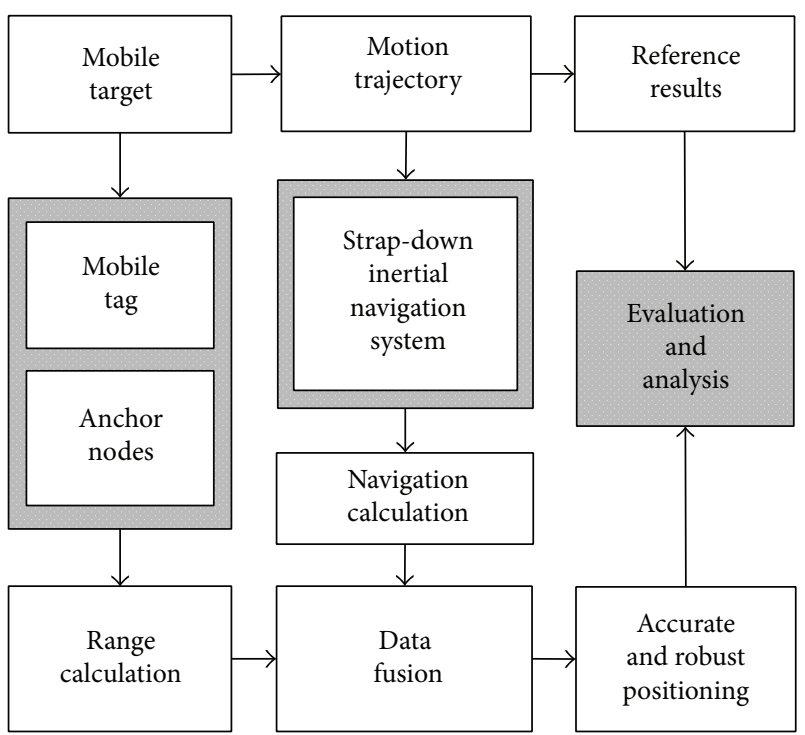

FIGURE 1: Block diagram of SINS and WSN.

TABLE 1: Technical parameters of SINS core component.

\begin{tabular}{lcc}
\hline ADIS16350 & Accelerometers & Gyroscopes \\
\hline Axes & 3 & 3 \\
Full Scale & $\pm 10 \mathrm{~g}$ & $\pm 75, \pm 150, \pm 300^{\circ} / \mathrm{s}$ \\
Bandwidth & $350 \mathrm{~Hz}$ & $350 \mathrm{~Hz}$ \\
Resolution Ratio & $14 \mathrm{bit}$ & $14 \mathrm{bit}$ \\
Sampling period & $0.01 \mathrm{~s}$ & $0.01 \mathrm{~s}$ \\
Baud rate & $115200 \mathrm{bit} / \mathrm{s}$ & $115200 \mathrm{bit} / \mathrm{s}$ \\
\hline
\end{tabular}

TABLE 2: Technical parameters of XBee.

\begin{tabular}{lc}
\hline XBee Pro S2b & XBee \\
\hline Operating frequency band & ISM $2.4 \mathrm{GHz}$ \\
Radius & $3.2 \mathrm{~km}$ \\
RF data rate & $250 \mathrm{kbps}$ \\
Idle current & $15 \mathrm{~mA}$ \\
Receiver sensitivity & $-102 \mathrm{dBm}$ \\
Supply voltage & $2.7 \mathrm{~V}-3.6 \mathrm{~V}$ \\
\hline
\end{tabular}

information. These measured parameters are used for subsequent computation and analysis based on proposed positioning method.

Figure 2 gives SINS method integrated with WSN method for the position of mobile target in detail. Firstly the TAA and TAG sensors of SINS instrument are used to measure the acceleration and angular velocity values; the attitude matrix can be deduced based on the initial velocity and location of mobile target. Considering that the mobile target is a three-dimensional carrier, the pose of mobile target has a great effect on the location accuracy. Hence doing some standardized treatments and coordinate transformations for attitude matrix, the processed attitude matrix and acceleration make vector coupling. Then the location of mobile target by SINS method can be obtained after double integral solution. Secondly the anchor nodes and mobile tag are utilized to measure the TOA values. Then the TDOA values are calculated based on the measured TOA values. The anchor nodes clocks need to be synchronized using the TDOA approach. Then the distances measurements among anchor nodes and mobile tag can be calculated based on the TDOA values. Thirdly, the positioning result is estimated by using the measured parameters by SINS and WSN methods.

\section{Positioning Technology}

3.1. SINS Positioning Approach. Figure 3 shows the actual object of SINS and XBee, while Tables 1 and 2 show the parameters for core component of SINS and XBee, respectively [21]. The SINS mainly consists of core module ADIS16350, signal processing module TMS320C6713 DSP, communication module MAX3111E, reset module ADM708T, power module MIC4722YML, and memory expansion module TMS320C6713BPYP200. The ADIS16350 module with inertial measurement unit of 6 degrees freedom measures three-axis accelerations and three-axis angular rates. The

ADIS16350 module is sufficiently stable in terms of gyro bias stability to detect the angular rates. The full scale of gyroscope can be adjusted from $\pm 75^{\circ} / \mathrm{s}, \pm 150^{\circ} / \mathrm{s}$ to $\pm 300^{\circ} / \mathrm{s}$, while its resolution ratio is 14 bit. Different from the gyroscopes, the accelerometers cannot always keep accurate measurement after a long time monitoring. The full scale of accelerometers is designed as $\pm 10 \mathrm{~g}$, while its resolution ratio is $14 \mathrm{bit}$. The ADIS16350 module uses RS-232 signaling protocol to transmit measured data at $115200 \mathrm{bit} / \mathrm{s}$ baud rate. The ADIS16350 module can effortlessly complete the positioning task of mobile target.

Due to the maneuvering state, the parameters of SINS transmitted to the computer through wired connection will limit the flexibility of mobile target. Hence, the XBee Pro S2b module is used to connect the SINS with computer via wireless communication model. The XBee Pro S2b module adopts a universal operating frequency band with ISM 2.4 GHz. The RF data rate is $250 \mathrm{kbps}$, while the maximum distance can be up to $3.2 \mathrm{~km}$. The XBee Pro S2b module is operated with about $-102 \mathrm{dBm}$ receiver sensitivity. The supply voltage is $2.7 \mathrm{~V}$ to $3.6 \mathrm{~V}$, and the idle current is about $15 \mathrm{~mA}$.

SINS approach is a dead reckoning system, whose accuracy degree depends on the previous time navigation parameter estimation. Hence, the initialization of inertial navigation system, such as the initial pose, velocity, and location is the basis of accurate estimation. Figure 4 describes the diagram of SINS mechanization arrangement for mobile target. SINS measures the acceleration and angular velocity values. The measured angular velocity values and initial angular velocity values are utilized to perform navigation calculation under Gravity compensation and Coriolis Theorem. Moreover, the navigation equation is converted and decomposed on east, north, and upper directions. According to the initial velocity and location, the position of mobile target by SINS approach can be calculated after performing quadratic integrations. 


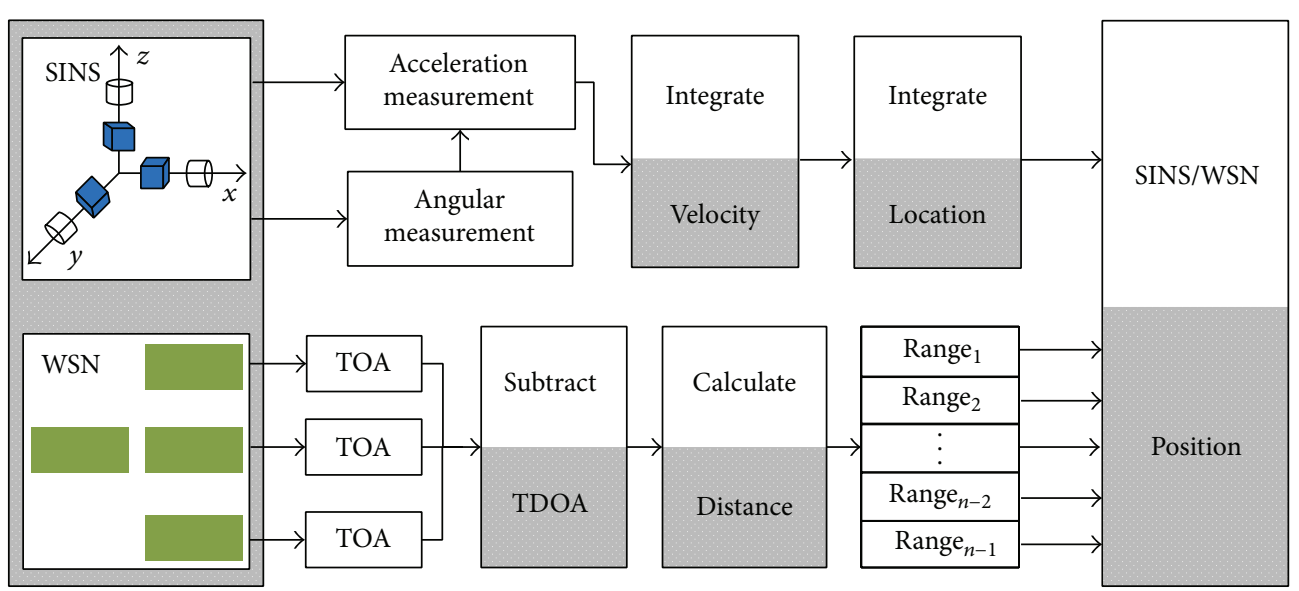

FIGURE 2: SINS integrated with WSN measurements for mobile target positioning.

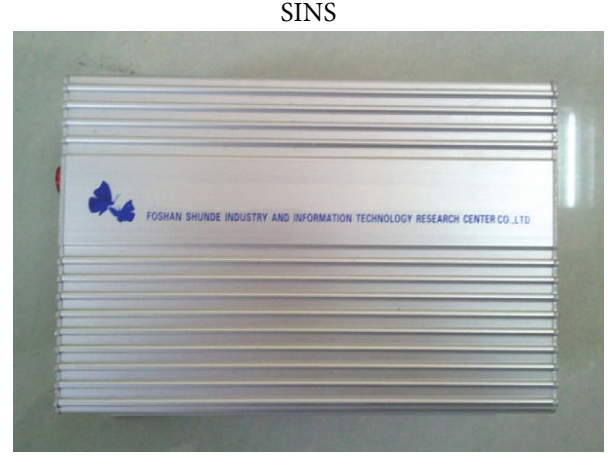

(a)

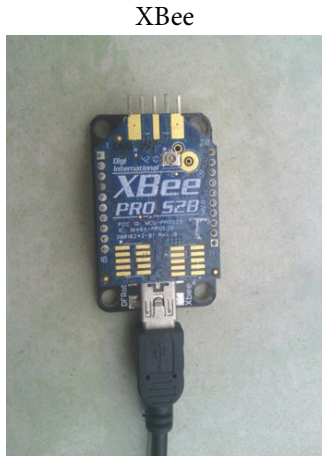

(b)

FIGURE 3: SINS and XBee: (a) SINS instrument and (b) XBee communication module.

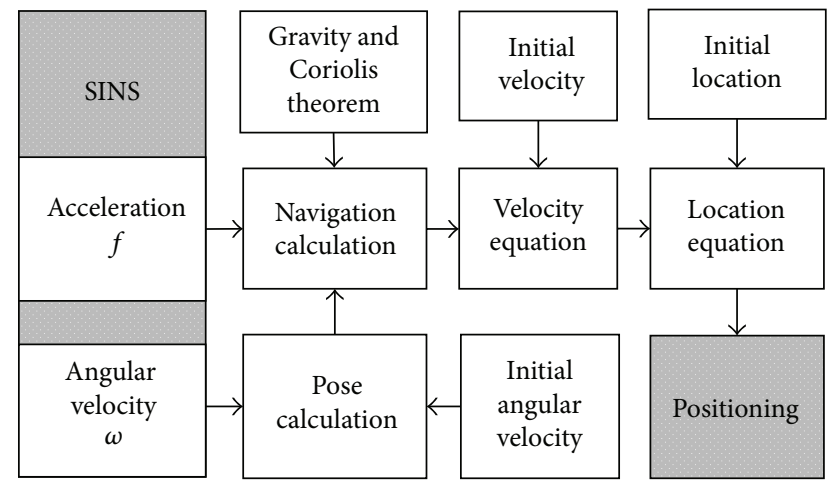

FIGURE 4: SINS mechanization arrangement for mobile target.

Supposing that SINS instrument is installed on mobile target, the quality of mobile target is $m_{g}$. Based on Newtonian mechanics theorem, the mechanical equation yields

$$
\mathbf{F}=\mathbf{F}_{\text {non- } g}+\mathbf{F}_{g}=m_{g} \frac{d^{2} \mathbf{R}}{d t^{2}}
$$

where $\mathbf{F}_{g}$ is the gravity force, $\mathbf{F}_{\text {non- } g}$ is the nongravity force, and $\mathbf{R}$ is the earth radius.
The speed of mobile target relative to the earth is defined as $\mathbf{V}_{e}$. Based on the pose matrix $\mathbf{C}_{b}^{n}$, we can obtain $[22,23]$

$$
\mathbf{f}^{n}=\dot{\mathbf{V}}_{e}^{n}+\left(2 \boldsymbol{\omega}_{i e}^{n}+\boldsymbol{\omega}_{e n}^{n}\right) \times \mathbf{V}_{e}^{n}-\mathbf{g}^{n}
$$

In Formula (2), the vector is decomposed as

$$
\begin{gathered}
\mathbf{f}^{n}=\left(f_{E}, f_{N}, f_{U}\right)^{T} \\
\mathbf{V}_{e}^{n}=\left(V_{E}, V_{N}, V_{U}\right)^{T} \\
\boldsymbol{\omega}_{i e}^{n}=\left(0, \omega_{i e} \cos L, \omega_{i e} \sin L\right)^{T} \\
\boldsymbol{\omega}_{e n}^{n}=\left(-\frac{V_{N}}{R_{M}+h}, \frac{V_{E}}{R_{N}+h}, \frac{V_{E}}{R_{N}+h} \tan L\right)^{T} \\
\mathbf{g}^{n}=(0,0,-g)^{T},
\end{gathered}
$$

where $\boldsymbol{\omega}_{i e}$ is the rotating angular velocity of inertial coordinate relative to the earth coordinate; $\boldsymbol{\omega}_{\text {en }}$ is the rotating angular velocity of navigation coordinate relative to the earth coordinate; $L$ is the local latitude; $R_{M}$ is the curvature radius of local meridian; $R_{N}$ is the curvature radius of prime vertical; and $h$ is the height of mobile target from the ground. 


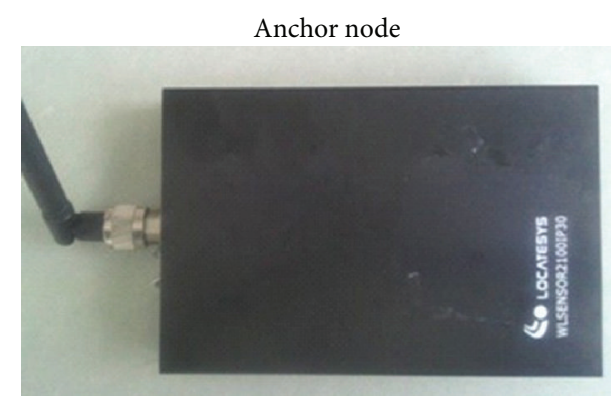

(a)

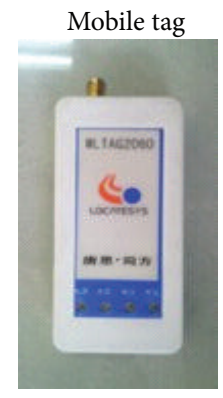

(b)

FIgURE 5: Anchor node and mobile tag: (a) anchor node and (b) mobile tag.

Applying Formulas (2) and (3), we can get

$$
\left(\begin{array}{c}
\dot{V}_{E} \\
\dot{V}_{N} \\
\dot{V}_{U}
\end{array}\right)=\left(\begin{array}{c}
f_{E} \\
f_{N} \\
f_{U}
\end{array}\right)-\left(\begin{array}{ccc}
0 & -\left(2 \omega_{i e} \sin L+\frac{V_{E} \tan L}{R_{N}+h}\right) & 2 \omega_{i e} \cos L+\frac{V_{E}}{R_{N}+h} \\
2 \omega_{i e} \sin L+\frac{V_{E} \tan L}{R_{N}+h} & 0 & \frac{V_{N}}{R_{M}+h} \\
-\left(2 \omega_{i e} \cos L+\frac{V_{E}}{R_{N}+h}\right) & -\frac{V_{N}}{R_{M}+h}
\end{array}\right)\left(\begin{array}{c}
V_{E} \\
V_{N} \\
V_{U}
\end{array}\right)+\left(\begin{array}{c}
0 \\
0 \\
-g
\end{array}\right)
$$

Substituting the initial velocity and location of mobile target into Formula (4), we can calculate and acquire the parameters of mobile target. Meanwhile considering that the mobile target moves around on the XOY plane, the effect of velocity $\dot{V}_{U}$ can be ignored. Hence, Formula (4) can be written as [23]

$$
\begin{gathered}
\dot{V}_{E}=f_{E}+2 V_{N} \omega_{i e} \sin L+\frac{V_{E} V_{N}}{R_{N}+h} \tan L \\
\dot{V}_{N}=f_{N}-2 V_{E} \omega_{i e} \sin L-\frac{V_{E}^{2}}{R_{N}+h} \tan L .
\end{gathered}
$$

From Formula (5), the position of mobile target can be calculated by performing quadratic integration. The navigation equations by SINS are used to establish equations. But the approximated results by pure SINS approach can be affected by multinoises, which will cause accumulative errors after a long time tracking. In the next section, WSN measurement approach is utilized to eliminate the accumulative errors by SINS approach.

3.2. WSN Positioning Approach. Figure 5 shows the actual object of anchor nodes and mobile tag, while Table 3 shows the parameters for core component of wireless nodes. The core component is the nanoLOC_TRX model provided by Nanotron technology. The wireless nodes mainly work in a unique communication technology defined as chirp spread spectrum (CSS) technique, which is specified at IEEE 802.15.4a in the ISM $2.4 \mathrm{GHz}$ frequency. The carrier sense multiple access/collision avoidance (CSMA/CA) and time division multiple access (TDMA) are supported. Furthermore, the radio transmission data rate can be adjusted from $125 \mathrm{k}$ bps to $2 \mathrm{Mbps}$, while the maximum distance depends on the data rate. The wireless nodes support the allowed $\mathrm{RF}$ output power from $-33 \mathrm{dBm}$ to $0 \mathrm{dBm}$ with the receiver sensitivity of $-95 \mathrm{dBm}$. The current value is about $1.2 \mu \mathrm{A}$ with minimal power consumption. The voltage $2.5 \mathrm{~V}$ is provided with $\pm 0.2 \mathrm{~V}$ drift. The wireless nodes adopt highly integration signal chips which can provide high wireless communication performance and accurate range measurement.

A few anchor nodes are placed with initial coordinates, written as the vector $\mathbf{a}=\left(\mathbf{a}_{1}, \mathbf{a}_{2}, \ldots, \mathbf{a}_{n}\right)^{T}$, where $\mathbf{a}_{i}=\left(x_{i}, y_{i}\right)^{T}$. The mobile tag is defined as $\mathbf{x}_{k}=\left(x_{k}, y_{k}\right)^{T}$. The WSN measurement approach is shown in Figure 6.

The estimation result by WSN is usually accomplished under two steps. Firstly, some wireless signals among mobile tag and anchor nodes are obtained to represent distance relationships. Secondly, the position of mobile target is estimated by solving a set of equations defined by the wireless signal parameters [24]. The principle of wireless distance measurement includes two independent processes, which can be described as shown in Figure 7.

The first distance measurement: the anchor node sends data stream to mobile tag and then receives the response from mobile tag. The anchor node calculates the transmission time $\tau_{1}$ from sending data stream to receiving data stream. Meanwhile, the mobile tag starts the clock when it receives data stream from anchor node and then stops the clock after sending the response to the anchor node. The mobile tag calculates the processing time $\tau_{2}$ and then sends it to anchor node. The second distance measurement: the mobile 
TABLE 3: Technical parameters of wireless node core component.

\begin{tabular}{lc}
\hline nanoLOC_TRX & Wireless nodes \\
\hline Number & 4 \\
ISM band & $2.4 \mathrm{GHz}$ \\
Update rata & $125 \mathrm{kbps}-2 \mathrm{M} \mathrm{bps}$ \\
Modulation & Chirp Spread Spectrum \\
RF output power & $-33 \mathrm{dBm}$ to $0 \mathrm{dBm}$ \\
Receive sensitivity & $-95 \mathrm{dBm}$ \\
Current value & $1.2 \mu \mathrm{A}$ \\
Supply voltage & $2.5 \mathrm{~V} \pm 0.2 \mathrm{~V}$ \\
\hline
\end{tabular}

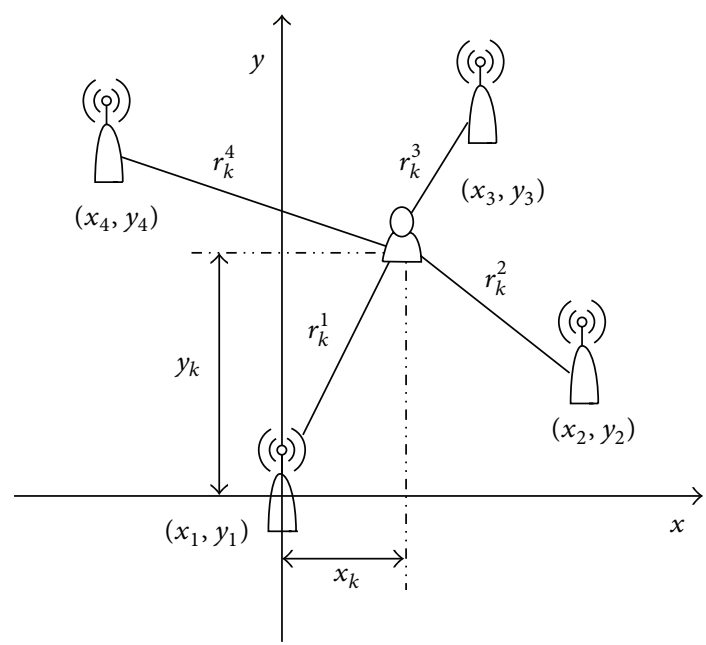

FIGURE 6: WSN measurement approach.

tag sends data stream to anchor node and then receives the response from anchor node. The mobile tag calculates the transmission time $\tau_{3}$ from sending data stream to receiving data stream and then sends the transmission time $\tau_{3}$ to anchor node. Meanwhile, the anchor node starts the clock when it receives data stream from mobile tag and then stops the clock after sending the response to the mobile tag. The anchor node calculates the processing time $\tau_{4}$. Then the distance between anchor node and mobile tag can be obtained:

$$
r_{k}^{i}=v_{c} t_{k}^{i}=v_{c} \frac{\tau_{1}-\tau_{2}+\tau_{3}-\tau_{4}}{4},
$$

where $r_{k}^{i}$ is the distance between anchor node and mobile tag; $v_{c}$ is the propagation speed of wireless signal, commonly defined as $3 \times 10^{8} \mathrm{~m} / \mathrm{s} ; t_{k}^{i}$ is the mean transmission time of actual measurement; $\tau_{1}, \tau_{2}, \tau_{3}$, and $\tau_{4}$ are the measurement times among anchor nodes and mobile tag.

Considering that the measurement deviations are caused by crystal oscillators, the measurement deviations of transmission times $\tau_{1}, \tau_{2}, \tau_{3}$, and $\tau_{4}$ are set as $\delta_{1}, \delta_{2}, \delta_{3}$, and $\delta_{4}$ respectively. Then distance measurement error can be described as

$$
\Delta r_{k}^{i}=v_{c} \frac{\delta_{1}-\delta_{2}+\delta_{3}-\delta_{4}}{4}
$$

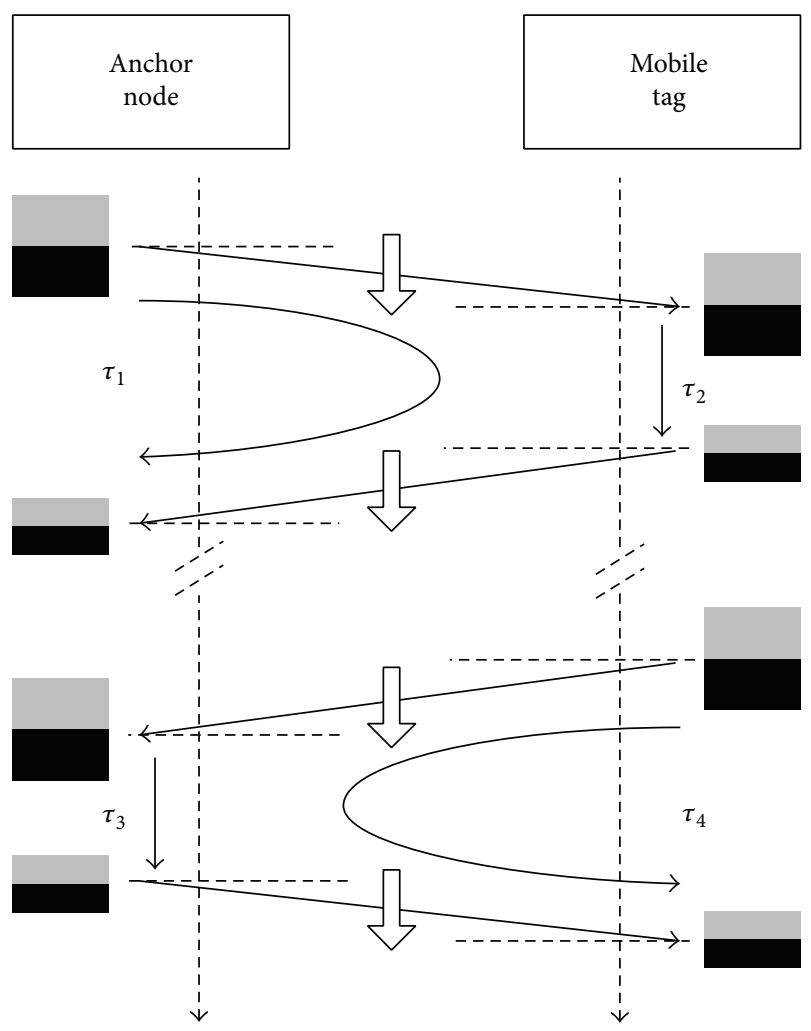

FIGURE 7: The principle of distance measurement.

The deviations $\delta_{1}$ and $\delta_{4}$ are caused by the crystal oscillator of anchor node, and the deviations $\delta_{2}$ and $\delta_{3}$ are caused by the crystal oscillator of mobile tag. Hence, the deviation $\delta_{4}$ is equal to the deviation $\delta_{1}$ and the deviation $\delta_{3}$ is equal to the deviation $\delta_{2}$. Then

$$
\delta_{1}=\delta_{4} ; \quad \delta_{2}=\delta_{3}
$$

Formula (7) can be expressed by

$$
\Delta r_{k}^{i}=v_{c} \frac{\delta_{1}-\delta_{2}+\delta_{2}-\delta_{1}}{4}=0 .
$$

The principle of distance measurement by the wireless chips can eliminate the measurement deviations caused by crystal oscillators. Then the distance $r_{k}^{i}$ between mobile tag and anchor node is

$$
r_{k}^{i}=\sqrt{\left(x_{i}-x_{k}\right)^{2}+\left(y_{i}-y_{k}\right)^{2}}, \quad i=1,2,3, \ldots, n .
$$

Formula (10) can be represented as the 2-norm:

$$
r_{k}^{i}=\left\|\mathbf{a}_{i}-\mathbf{x}_{k}\right\|, \quad i=1,2,3, \ldots, n .
$$

The TDOA value with respect to the first anchor node is denoted as $r_{k}^{t, 1}$. Then the TDOA distance yields

$$
r_{k}^{i, 1}=r_{k}^{i}-r_{k}^{1}=\left\|\mathbf{a}_{i}-\mathbf{x}_{k}\right\|-\left\|\mathbf{a}_{1}-\mathbf{x}_{k}\right\|, \quad i=2,3, \ldots, n .
$$


The TDOA value from the mobile tag can be represented as the vector $\mathbf{r}_{k}=\left(r_{k}^{2,1}, r_{k}^{3,1}, \ldots, r_{k}^{n, 1}\right)^{T}$, which can be expressed as

$$
\mathbf{r}_{k}=\left(\begin{array}{c}
\sqrt{\left(x_{2}-x_{k}\right)^{2}+\left(y_{2}-y_{k}\right)^{2}}-\sqrt{\left(x_{1}-x_{k}\right)^{2}+\left(y_{1}-y_{k}\right)^{2}} \\
\sqrt{\left(x_{3}-x_{k}\right)^{2}+\left(y_{3}-y_{k}\right)^{2}}-\sqrt{\left(x_{1}-x_{k}\right)^{2}+\left(y_{1}-y_{k}\right)^{2}} \\
\vdots \\
\sqrt{\left(x_{n}-x_{k}\right)^{2}+\left(y_{n}-y_{k}\right)^{2}}-\sqrt{\left(x_{1}-x_{k}\right)^{2}+\left(y_{1}-y_{k}\right)^{2}}
\end{array}\right) .
$$

From Formulas (6) and (13), the position of mobile target by WSN method is approximated. The unpredicted environments may make wireless signal suffer from multicomplex noises. The accuracy of wireless signals is sensitive to the environment conditions. Hence combining the above SINS method with WSN method, a novel and effective fusion method needs to be utilized to create an optimal positioning estimation.

\subsection{Pure SINS and WSN Experiments. The monitoring area} is about $12 \times 7 \mathrm{~m}^{2}$. The SINS and mobile tag are installed on an electric car that is defined as mobile target. The detail conditions of positioning system are given as [21]: the earth spin velocity is $7.2916 \times 10^{-5} \mathrm{rad} / \mathrm{s}$; the local latitude is $34.316^{\circ}$. The earth gravitational acceleration is $9.7952 \mathrm{~m} / \mathrm{s}^{2}$, and the gravitational acceleration accuracy is $2 \times 10^{-5} \mathrm{~g}$. Owing to the fact that the electric car moves on the flat ground, the initial angular values are zero. The initial velocity of electric car is zero, and the coordinate of initial location is $(6,1.8) \mathrm{m}$. Furthermore, four anchor nodes are deployed in the corners. The coordinates of anchor nodes are $(1.3,1.6)$ $\mathrm{m},(10.8,1.6) \mathrm{m},(10.8,5.4) \mathrm{m}$, and $(1.3,5.4) \mathrm{m}$, respectively. The positioning measurements are carried out in an office environment under line of sight (LOS) conditions. For the first set of experiments, we evaluate the estimation results under pure SINS and WSN approaches.

The electric car is equipped with the infrared sensor, which makes the electronic car move along the black insulating tape. Then we can measure the trajectory of the black line. The electric car moves around and forms a projected trajectory in curve, which can be used to evaluate and analyze the performance of nonlinear system using the proposed positioning method.

For the first set of experiments, we evaluate the estimations results using pure SINS and WSN methods. As shown in Figure 8, the estimation results reveal that the pure SINS and WSN approaches have approximately stable positioning performance regardless of the mobile target mobility. When the mobile target is in the beginning motion, the SINS approach has the excellent estimation. As long as making some appropriate initial settings, such as the initial pose, velocity, and location, the SINS approach can obtain an accurate result. However with a long time monitoring, the mobile target by SINS approach can break away from the projected trajectory due to multinoises. No matter where the mobile target moves, the WSN approach always has a certain estimation error.

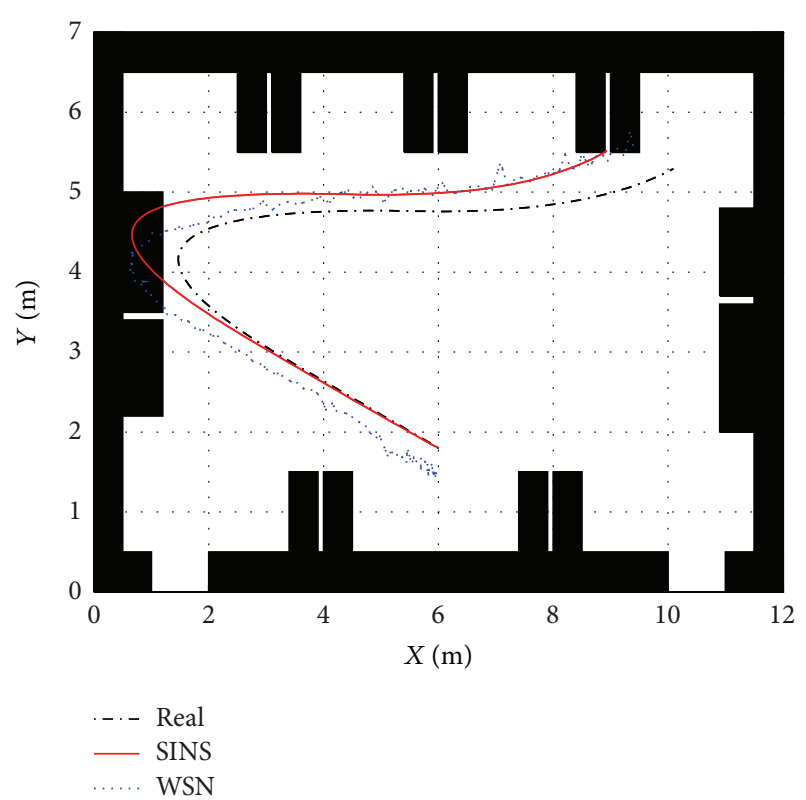

FIgURE 8: Positioning tracking of pure SINS and WSN approaches.

Figure 9 compares the estimation errors of $X$ - and $Y$-axis under pure SINS and WSN approaches. Experimental results indicate that the estimation error of $X$-axis by SINS and WSN approaches is larger than the error of $Y$-axis. This is mainly because the motion range of mobile target along $X$-axis is more expansive than that of the $Y$-axis, which makes that the WSN estimation errors are mainly distributed in the $X$-axis.

The mean estimation errors of SINS and WSN approaches are 0.69 and $0.64 \mathrm{~m}$, respectively. The variance errors of SINS and WSN approaches are 0.53 and 0.18 , respectively. As expected, the overall trend of positioning errors by SINS approach rises with time. The positioning error of SINS method begins to increase after turning a corner and keeps a sharp increasing trend. Overall, the SINS has the maximum error $1.27 \mathrm{~m}$ around the end of sampling point 125 for its accumulative error over time.

Meanwhile, the positioning error of WSN approach increases firstly and then decreases when the mobile target begins to turn a corner. In addition to the distance measurement accuracy of wireless signals, the geometric relationship among mobile tag and anchor nodes also affects the positioning accuracy. When the mobile target begins to turn a corner, the mobile tag will start to move near the anchor nodes, which can cause the larger estimation errors, in comparison with the situation when the mobile tag moves in the center of monitoring areas. Hence, the maximum and minimum estimation errors of pure WSN are $0.94 \mathrm{~m}$ and $0.23 \mathrm{~m}$, respectively.

In addition to the position estimation, the SINS has three-axis gyroscope (TAG), which can be used for estimating the pose of mobile target. Based on implementing the SINS initial alignment, we begin to test the pitch, roll, and yaw angles. Figure 10 provides the estimation errors of pitch, roll, and yaw angles during the sampling time. The three-axis gyroscope by MEMS technology can provide 

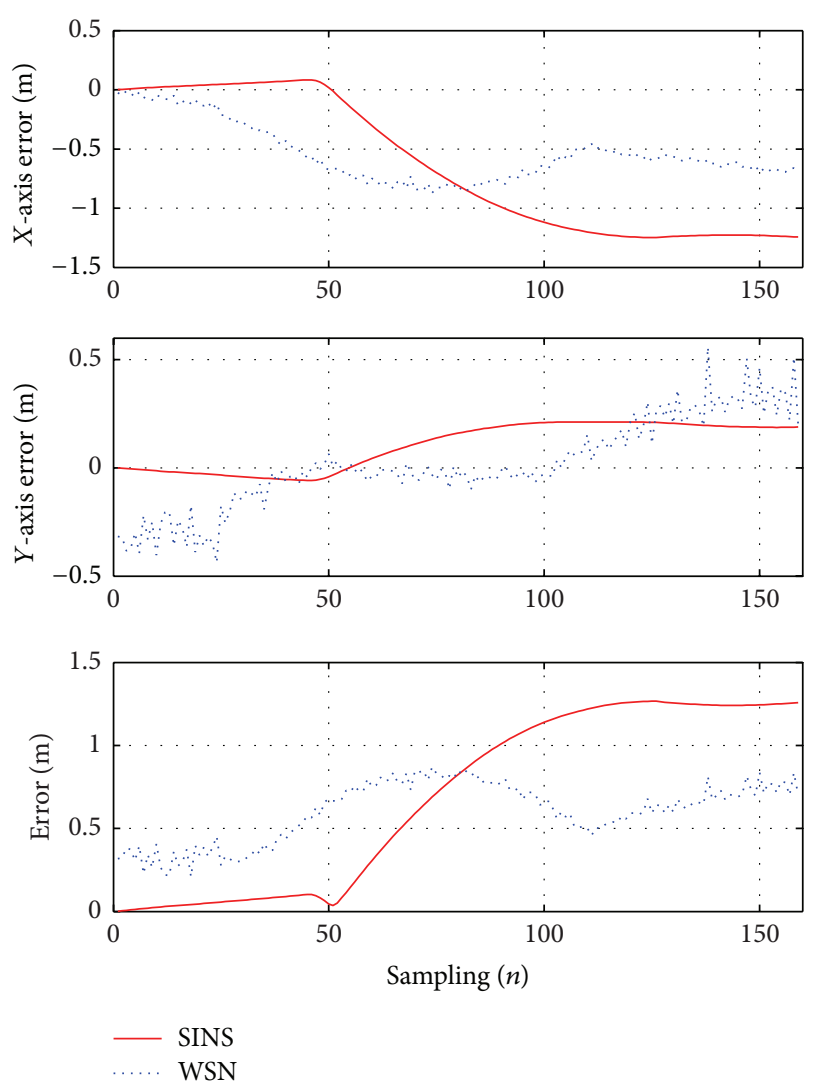

FIGURE 9: Estimation performances under pure SINS and WSN approaches.

high measurement performance. The mean estimation errors of pitch, roll, and yaw angles are $-0.6790,-0.1913$, and $-0.2370 \mathrm{deg}$, respectively. The measurement errors are mainly caused by initial alignment and random noises. Different from the positioning estimation, the pose estimation has no cumulative errors by pure SINS approach.

From Figure 10, we can conclude that the pure SINS can provide accurate pose estimation, whose accuracy needs not be improved. However from Figures 8 and 9, we can conclude that the pure SINS and WSN approaches cannot provide the accurate and robust positioning accuracy. Hence, the SINS/WSN integration is performed to improve the positioning performance in the next section.

\section{Integrated SINS/WSN Approaches}

4.1. The Implementation of Proposed Approach. The fusion method significantly affects the accuracy and robustness of positioning system. The KF or EKF method has been applied to the SINS/WSN positioning systems. KF method uses the residuals of the acceleration, velocity, and position values by SINS method, integrated with the distance measurement by WSN method. KF method is valid for linear systems and has good positioning performance, while it has large estimation errors for nonlinear systems. Usually, the state equation and measurement equation are expressed with nonlinear
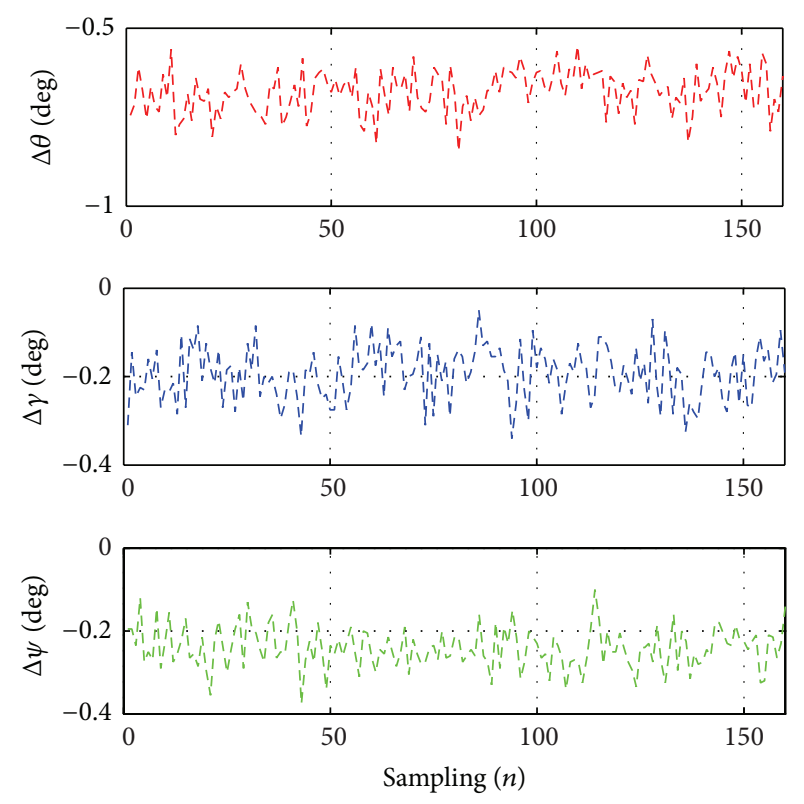

FIGURE 10: Estimation errors of pitch, roll, and yaw angles under SINS approach.

characteristic that makes the KF method breaks away from the guideline track and eventually diverges. EKF method can be used to estimate the nonlinear system, although EKF method still has certain estimation error with time.

The UKF method is designed to estimate position of mobile target by nonlinear state space models [25]. The accuracy of UKF method is up to the second order from Taylor series expansions of the associated nonlinear functions in contrast to the first order terms of EKF method, which has the same computational complexity as that of EKF method. The UKF method is applied with nonlinear state space models. The essence of UKF method can be briefly summarized as follows. A set of deterministically selected points, which are termed sigma points, is used to approximate the distribution of the previous state estimate. These sigma points are projected using the nonlinear measurement function. Moreover, it correctly captures the posterior mean and variance through the weighted mean and variance of the post. Then, accurate position can be estimated by UKF method [26].

Considering that mobile target moves around on XOY plane, the acceleration, velocity, and location are expressed as $\mathbf{x}_{k}=\left[x_{k}, y_{k}, \dot{x}_{k}, \dot{y}_{k}, \ddot{x}_{k}, \ddot{y}_{k}\right]^{T}$ at time step $k$. Defining the noise as $\mathbf{w}_{k}$, the state equations of mobile target can be given by [27]

$$
\mathbf{x}_{k+1}=\mathbf{f}_{k}\left(\mathbf{x}_{k}\right)+\mathbf{w}_{k}=\mathbf{f}_{k} \mathbf{x}_{k}+\mathbf{w}_{k} .
$$

At time step $k$, the mobile tag is installed on mobile target. The distance relationships among mobile tag and anchor nodes can be obtained to measure the range using the measurement values. Then defining the noise as $\mathbf{v}_{k}$, the measurement equations of mobile target can be given by

$$
\mathbf{z}_{k}=\mathbf{h}_{k}\left(\mathbf{x}_{k}\right)+\mathbf{v}_{k}
$$




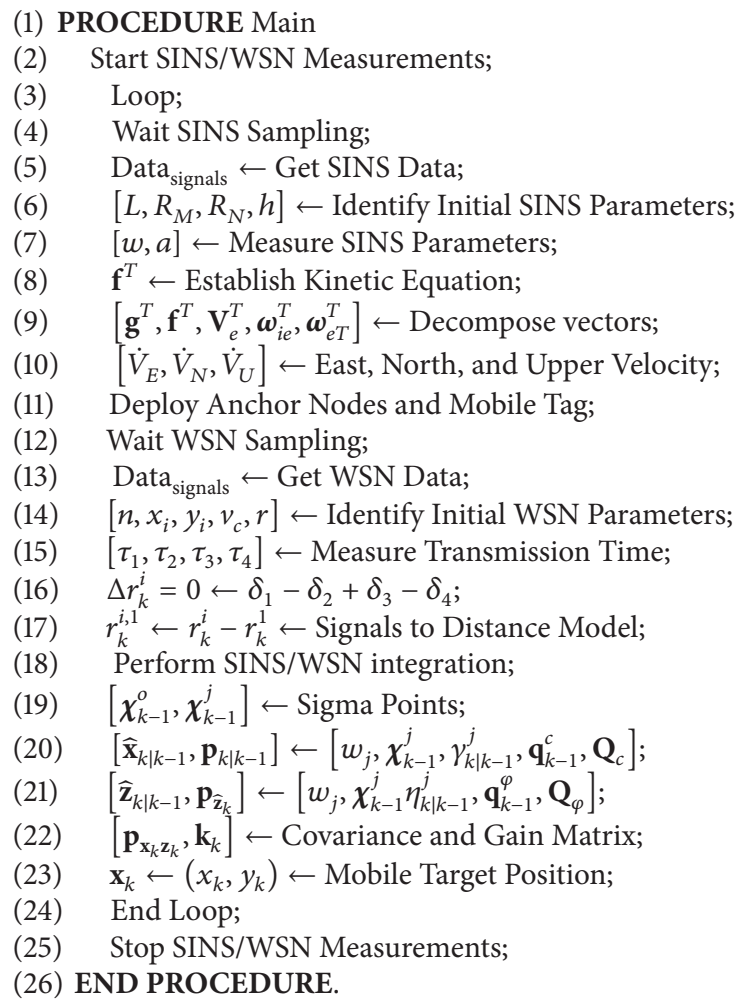

Algorithm 1: The flow of operations for the proposed positioning algorithm.

As for the random motion of mobile target, the positioning process is a nonlinear dynamic system. In the next section, the measurement parameters are acquired using SINS and WSN methods in order to establish the equations.

The UKF method is a straightforward extension to the recursive estimation, which has been applied in nonlinear system. Through creating state equations and measurement equations, the optimal estimation of mobile target can be obtained using UKF method. To give enough details of the implemented processes, we present algorithm pseudocode of the main program as shown in Algorithm 1.

Through the acquired measurement values of SINS and WSN approaches and initial parameters, the state equations and measurement equations can be created. Additionally, as noted above, the proposed method is a straightforward extension to the recursive estimation, which has been applied in nonlinear system. The optimal positioning results of mobile target are estimated using proposed method.

4.2. The Prototype of Experimental System. In this section, an experimental platform is implemented for evaluating and verifying the positioning system based on SINS and WSN approaches. Figure 11 shows the hardware connection diagram of positioning system. The designed system mainly consists of SINS (core component ADIS16350), anchor nodes (core component nanoLOC_TRX), mobile tag, XBee, router, and computer. The measured angle, velocity, and location by SINS are forwarded to computer through XBee connection.
The mobile tag communicates with anchor nodes, whose clocks have been synchronized using the wired connection. Then the measured wireless signals by WSN are transmitted to computer via the router as well. Moreover, all acquired parameters by SINS and WSN, such as angle, velocity, location, and wireless signals are sampled and analyzed in the computer.

The diagram of sensors and instruments deployment is illustrated in Figure 12. Its process of implementation is briefly described, together with the main sensors and instruments. The SINS, mobile tag, and XBee are installed on the mobile target. Four anchor nodes, another XBee, router, and computer are deployed in the monitoring area. Based on designing and solving the proposed algorithm, the position of mobile target can be produced in the computer. Then we can evaluate and verify the accuracy and robustness of proposed method.

4.3. SINS/WSN Integration Experiments. The tracking discrepancy of the KF, EKF, and UKF methods between the manually measured and estimated the trajectory can clearly be seen in Figure 13. We can conclude that the KF, EKF, and UKF methods can track the projected trajectory well when the mobile target moves before the fiftieth sampling time. This means that the SINS/WSN integration method can ameliorate the positioning performance. This behavior disappears after the fiftieth sampling time. As the mobile target turns a corner, the three methods fail to track well 


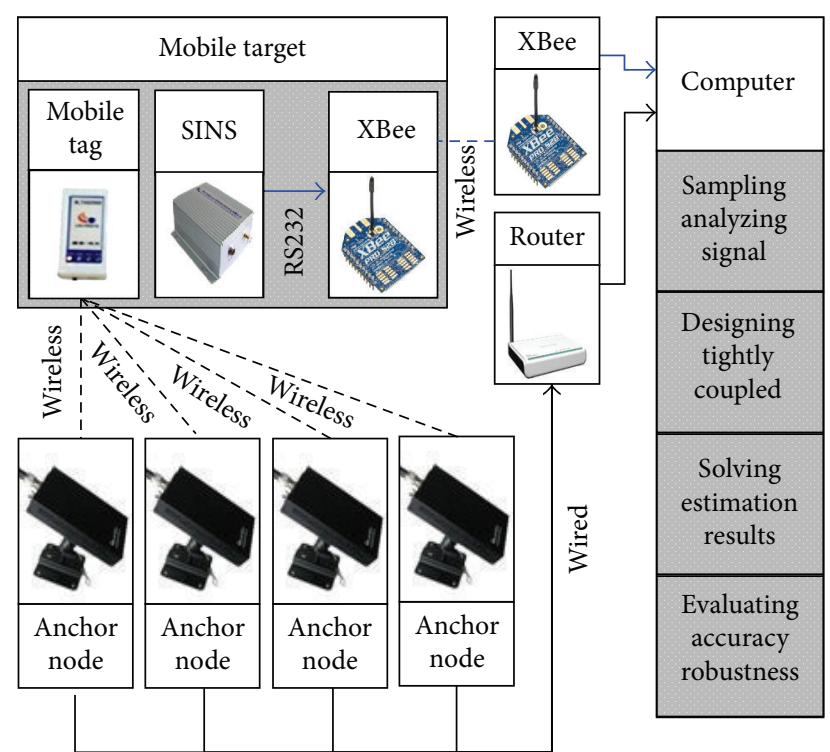

FIGURE 11: The hardware connection diagram of positioning system.

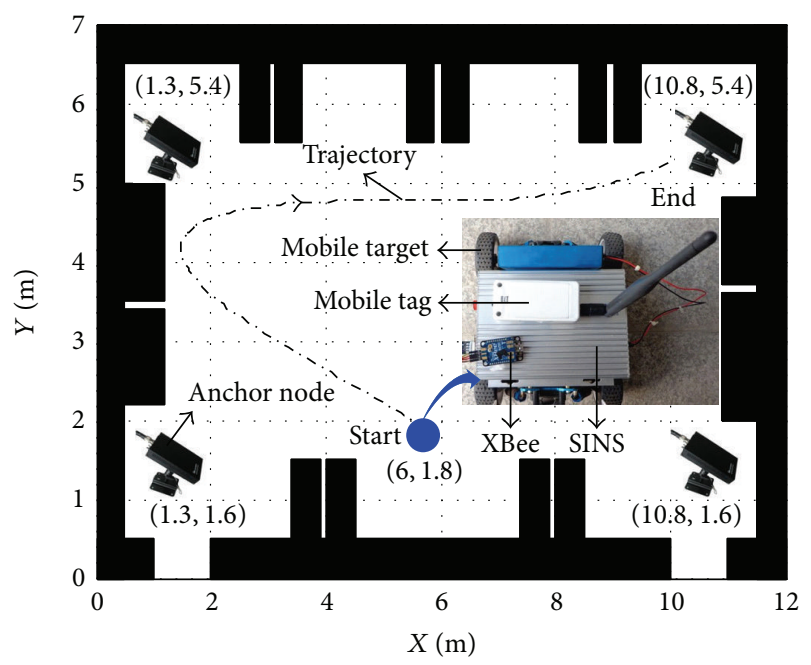

FIGURE 12: The sensors and instruments deployment diagram.

and have larger estimation errors until the end. Although it is difficult to measure the exact angle, velocity, location, and distance, the SINS/WSN integration based on UKF method, as in contrast to the pure SINS or WSN approach, can give more accurate positioning estimation.

Figure 14 describes the estimation errors of the KF, EKF, and UKF methods on the $X$ - and $Y$-axis. The position error on the $X$-axis is larger than the error on $Y$-axis due to the fact that the smaller acceleration values of $Y$-axis can make the mobile target in the limited motion range. The mean estimation errors of KF, EKF, and UKF methods are $0.71 \mathrm{~m}$, $0.63 \mathrm{~m}$, and $0.59 \mathrm{~m}$, respectively. The mobile target moves in the maneuvering motions and the SINS and WSN approaches have measurement noises, for which three methods have the increasing estimation errors with sampling time.

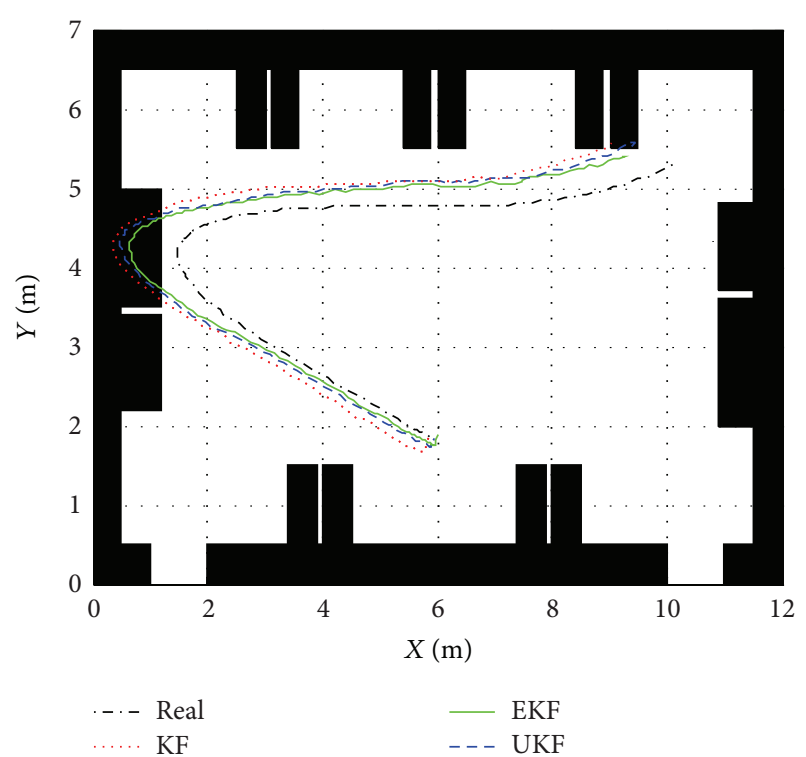

FIgURE 13: Positioning tracking of the KF, EKF, and UKF methods.
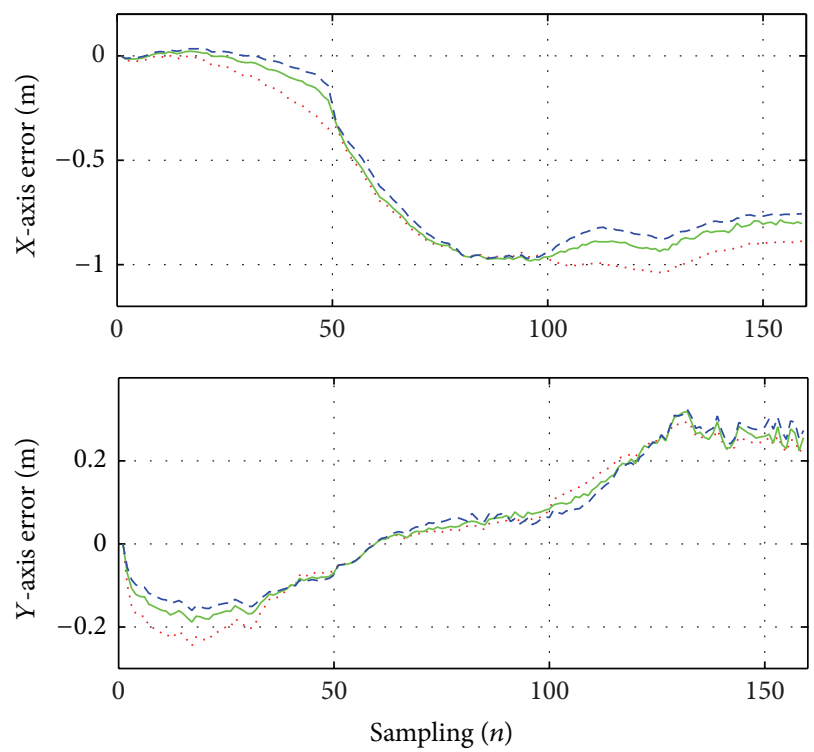

$\begin{array}{ll}\ldots . . . & \text { KF } \\ - & \text { EKF } \\ \text { - - } & \text { UKF }\end{array}$

FIGURE 14: Estimation performances of the SINS/WSN integration based on KF, EKF, and UKF methods.

Figure 15 lists estimation errors of multipositioning methods. The pure SINS method has the maximum error with $1.27 \mathrm{~m}$. Meanwhile, the pure WSN method has the maximum error with $0.94 \mathrm{~m}$ and minimum error with $0.23 \mathrm{~m}$. This is due to the maneuvering motions of mobile target and the noises measurement results of SINS and WSN methods. In comparison with the pure SINS, pure WSN, KF, and EKF methods, the mean estimation error of SINS/WSN integration based on the UKF method can reduce the errors 


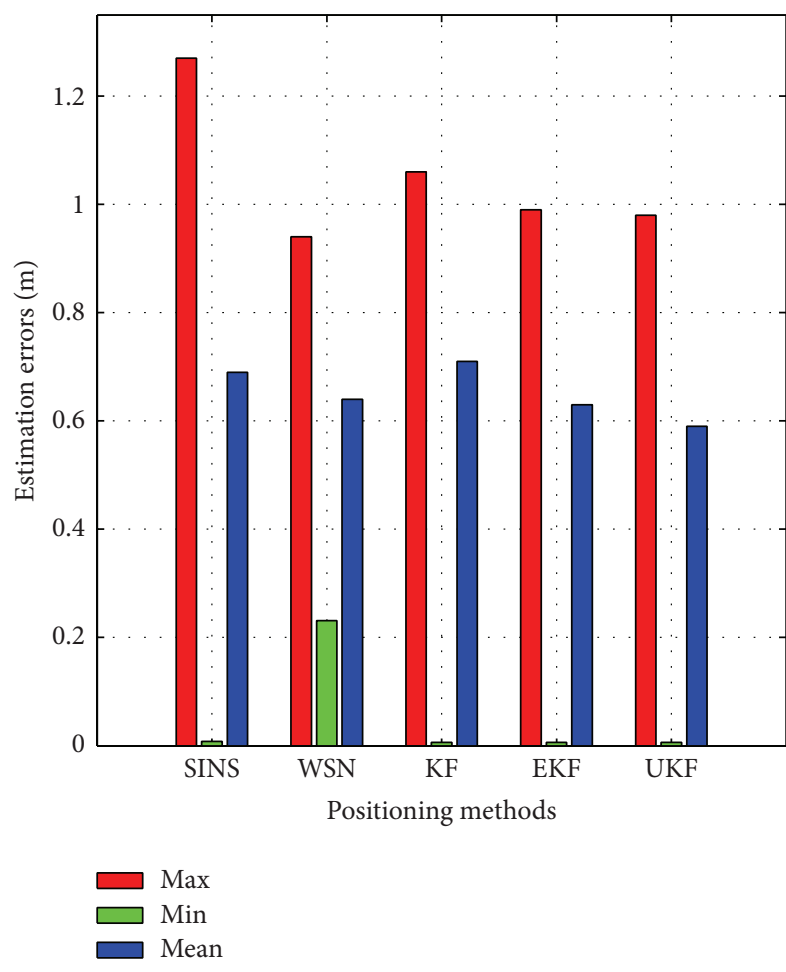

FIGURE 15: Estimation errors of multipositioning methods.

about $15 \%, 8 \%, 17 \%$, and $6 \%$, respectively, which indicates that UKF method can enhance the efficiency of SINS/WSN integration.

The proposed SINS/WSN integration based on the UKF method utilizes large hardware resources and increases the implementation complexity of communication and computation. However, there is no doubt that the SINS/WSN integration can bring more effective information and improve the accuracy and robustness of position estimation. Besides, the proposed method can realize synchronized position and pose tracking for mobile target, which can be applied in many applications.

\section{Conclusions}

This paper surveys the positioning technology for mobile target. Different positioning approaches present different estimation accuracy. Pure SINS or WSN positioning technology can be used to track the mobile target, while SINS approach has accumulative error after a long time tracking and WSN approach has anomalous error at certain monitoring points. The positioning technology using SINS and WSN integrated measurements is researched as the accurate and robust positioning is required in many engineering and science applications. The SINS/WSN integration is evaluated on the actual environment platform. The results indicate that the proposed method has complementary advantages of the SINS and WSN approaches and tracks the projected trajectory of mobile target well. In our future works, we will extend our researches on improving the measurement accuracy of SINS and WSN methods.

\section{Conflict of Interests}

The authors declare that there is no conflict of interests regarding the publication of this paper.

\section{Acknowledgments}

This work was done with support of China University of Mining and Technology. In addition, this work was supported by the National High Technology Research and Development Program of China (2013AA06A411), the Graduate Education Innovation Project of Jiangsu Province (CXZZ12_0925, KYLX_1374), and A Project Funded by the Priority Academic Program Development of Jiangsu Higher Education Institutions. The authors would like to thank the anonymous reviewers for their helpful comments which have improved the quality of the paper.

\section{References}

[1] B. S. Yaakov, Multitarget/Multisensor Tracking: Applications and Advances, Artech House, 1995.

[2] H. Liu, H. Darabi, P. Banerjee, and J. Liu, "Survey of wireless indoor positioning techniques and systems," IEEE Transactions on Systems, Man and Cybernetic C: Applications and Reviews, vol. 37, no. 6, pp. 1067-1080, 2007.

[3] C. M. Luo, W. Li, H. Yang, M. B. Fan, and X. F. Yang, "Mobile target positioning using refining distance measurements with inaccurate anchor nodes in chain-type wireless sensor networks," Mobile Networks and Applications, vol. 19, no. 3, pp. 363381, 2014.

[4] A. Noureldin, T. B. Karamat, and J. Georgy, Fundamentals of Inertial Navigation, Satellite-Based Positioning and Their Integration, Springer, 2013.

[5] Z. Lü, D. Liu, and C.-G. Li, “The precise positioning system of the mine personnel in coal pit based on strapdown inertial navigation," Journal of the China Coal Society, vol. 34, no. 8, pp. 1149-1152, 2009.

[6] F. Hoflinger, J. Muller, R. Zhang, L. M. Reindl, and W. Burgard, "A wireless micro inertial measurement unit (IMU)," IEEE Transactions on Instrumentation and Measurement, vol. 62, no. 9, pp. 2583-2595, 2013.

[7] R. Li, J. Georgy, J. Zhao, and A. Noureldin, "Testing a new integrated solution for MEMS inertial measurement unit used for measurement-while-drilling in rotary steerable system," Sensor Letters, vol. 10, no. 3-4, pp. 719-725, 2012.

[8] A. Morrison, V. Renaudin, J. B. Bancroft, and G. Lachapelle, "Design and testing of a multi-sensor pedestrian location and navigation platform," Sensors, vol. 12, no. 3, pp. 3720-3738, 2012.

[9] Y. Li, M. Efatmaneshnik, and A. G. Dempster, "Attitude determination by integration of MEMS inertial sensors and GPS for autonomous agriculture applications," GPS Solutions, vol. 16, no. 1, pp. 41-52, 2012.

[10] A. Boukerche, H. A. B. F. Oliveira, E. F. Nakamura, and A. A. F. Loureiro, "Localization systems for wireless sensor networks," IEEE Wireless Communications, vol. 14, no. 6, pp. 6-12, 2007.

[11] S. Mazuelas, A. Bahillo, R. M. Lorenzo et al., "Robust indoor positioning provided by real-time rssi values in unmodified WLAN networks," IEEE Journal on Selected Topics in Signal Processing, vol. 3, no. 5, pp. 821-831, 2009. 
[12] Y. T. Chan and K. C. Ho, "A simple and efficient estimator for hyperbolic location," IEEE Transactions on Signal Processing, vol. 42, no. 8, pp. 1905-1915, 1994.

[13] J.-A. Jiang, X.-Y. Zheng, Y.-F. Chen et al., "A distributed rssbased localization using a dynamic circle expanding mechanism," IEEE Sensors Journal, vol. 13, no. 10, pp. 3754-3766, 2013.

[14] Y. W. Zhang, A. K. Brown, W. Q. Malik, and D. J. Edwards, "High resolution 3-D angle of arrival determination for indoor UWB multipath propagation," IEEE Transactions on Wireless Communications, vol. 7, no. 8, pp. 3047-3055, 2008.

[15] M. R. Zoghi and M. H. Kahaei, "Sensor selection for target tracking in WSN using Modified INS algorithm," in Proceedings of the 3rd International Conference on Information and Communication Technologies: From Theory to Applications (ICTTA '08), pp. 1-6, Damascus, Syria, April 2008.

[16] Z. B. Zhu, X. B. Li, X. B. Li, and G. X. Qin, "Integration of inertial navigation system and wireless sensor network for mine environment," in Proceedings of the 2nd International Symposium on Test Automation and Instrumentation, pp. 18571860, Beijing, China, November 2008.

[17] C. Ascher, L. Zwirello, T. Zwick, and G. Trommer, "Integrity monitoring for UWB/INS tightly coupled pedestrian indoor scenarios," in Proceedings of the International Conference on Indoor Positioning and Indoor Navigation (IPIN '11), pp. 1-6, Guimar, Spain, September 2011.

[18] J. D. Hol, F. Dijkstra, H. Luinge, and T. B. Schöny, "Tightly coupled UWB/IMU pose estimation," in Proceedings of the IEEE International Conference on Ultra-Wideband, pp. 9-11, Vancouver, Canada, September 2009.

[19] A. de Angelis, J. O. Nilsson, I. Skog, P. Händel, and P. Carbone, "Indoor positioning by ultrawide band radio aided inertial navigation," Metrology and Measurement Systems, vol. 17, no. 3, pp. 447-460, 2010.

[20] K. Zhang, M. Zhu, G. Retscher, F. Wu, and W. Cartwright, "Three-dimension indoor positioning algorithms using an integrated RFID/INS system in multi-storey buildings," in Location Based Services and TeleCartography II, pp. 373-386, 2009.

[21] Q. G. Fan, W. Li, and C. M. Luo, "Error analysis and reduction for shearer positioning using the strapdown inertial navigation system," International Journal of Computer Science Issues, vol. 9, no. 5, pp. 49-54, 2012.

[22] M. Sotak, "Testing the coarse alignment algorithm using rotation platform," Acta Polytechnica Hungarica, vol. 7, no. 5, pp. 87107, 2010.

[23] Y. Bar-Shalom, X. R. Li, and T. Kirubarajan, Estimation with Applications to Tracking and Navigation, John Wiley \& Sons, New York, NY, USA, 2001.

[24] T. Jia and R. M. Buehrer, "On the optimal performance of collaborative position location," IEEE Transactions on Wireless Communications, vol. 9, no. 1, pp. 374-383, 2010.

[25] S. J. Julier and J. K. Uhlmann, A General Method for Approximating Nonlinear Transformations of Probability Distributions, Department of Engineering Science, University of Oxford, 1996.

[26] S.-M. Chow, E. Ferrer, and J. R. Nesselroade, "An unscented kalman filter approach to the estimation of nonlinear dynamical systems models," Multivariate Behavioral Research, vol. 42, no. 2, pp. 283-321, 2007.

[27] H. Song, V. Shin, and M. Jeon, "Mobile node localization using fusion prediction-based interacting multiple model in cricket sensor network," IEEE Transactions on Industrial Electronics, vol. 59, no. 11, pp. 4349-4359, 2012. 

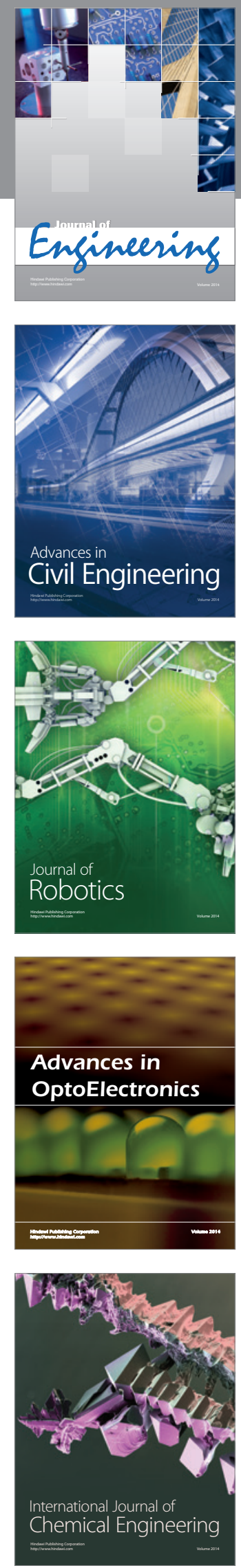

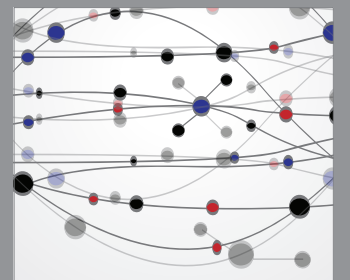

The Scientific World Journal
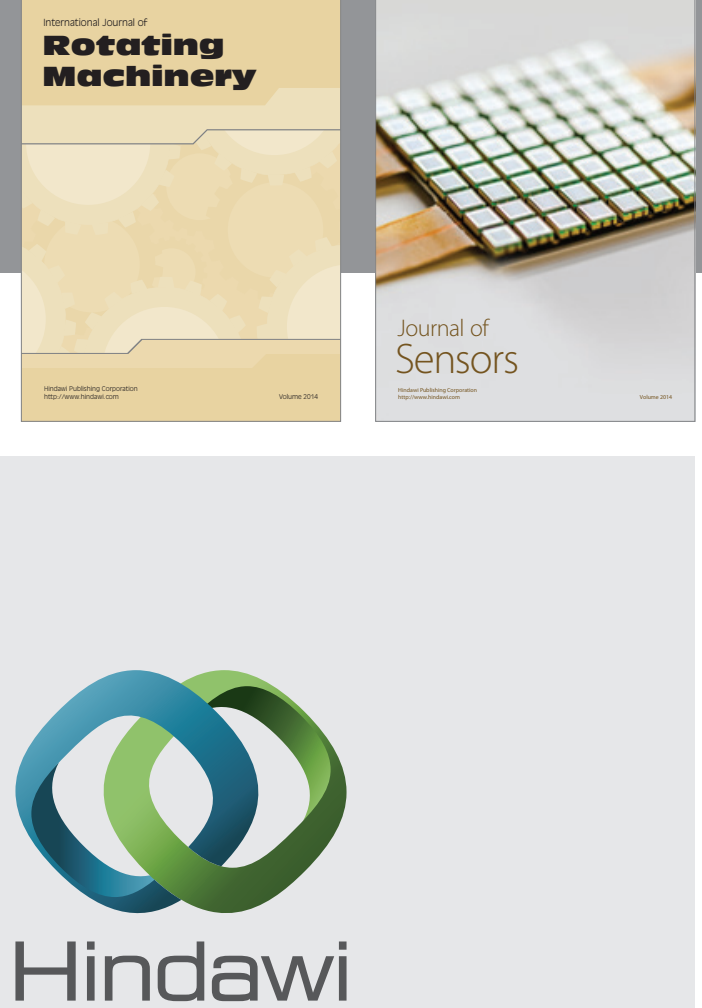

Submit your manuscripts at http://www.hindawi.com
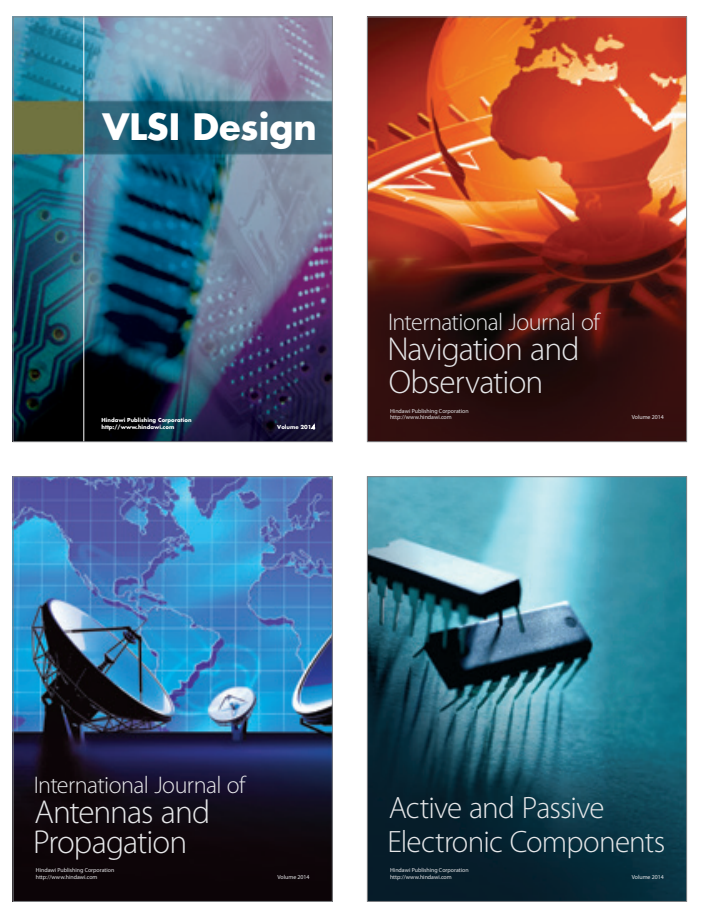
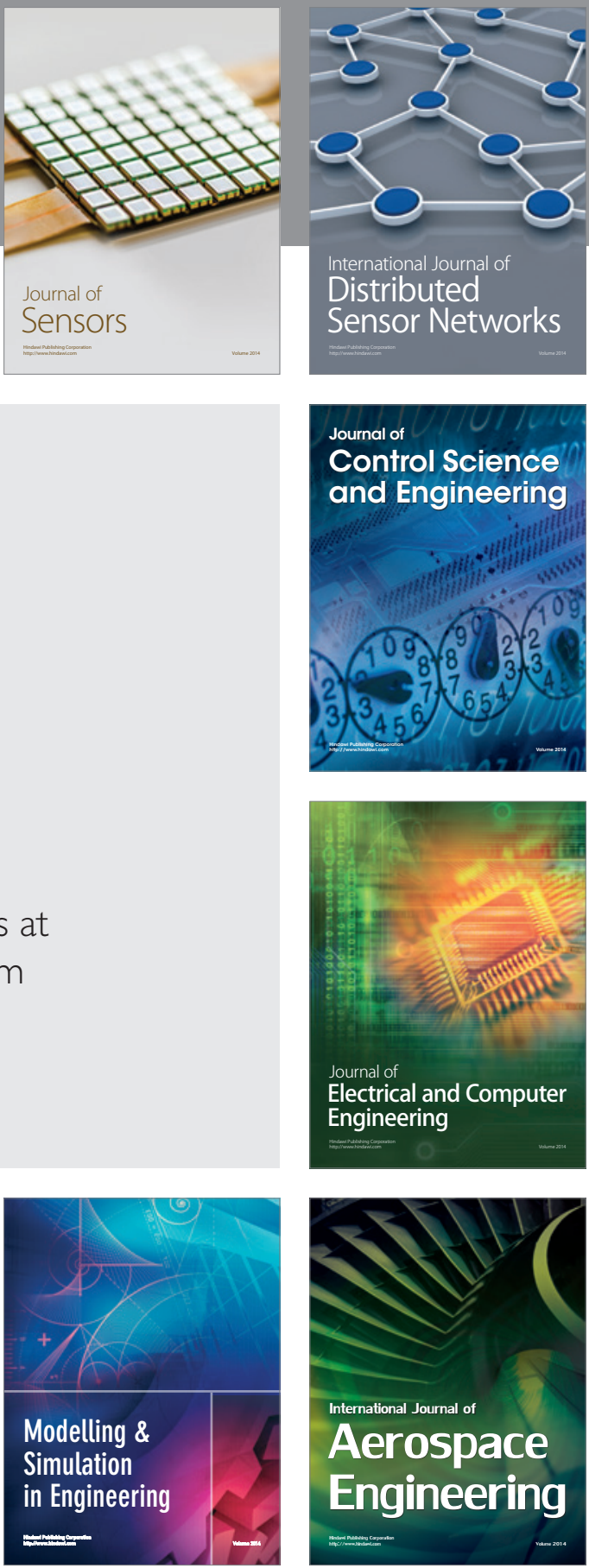

Journal of

Control Science

and Engineering
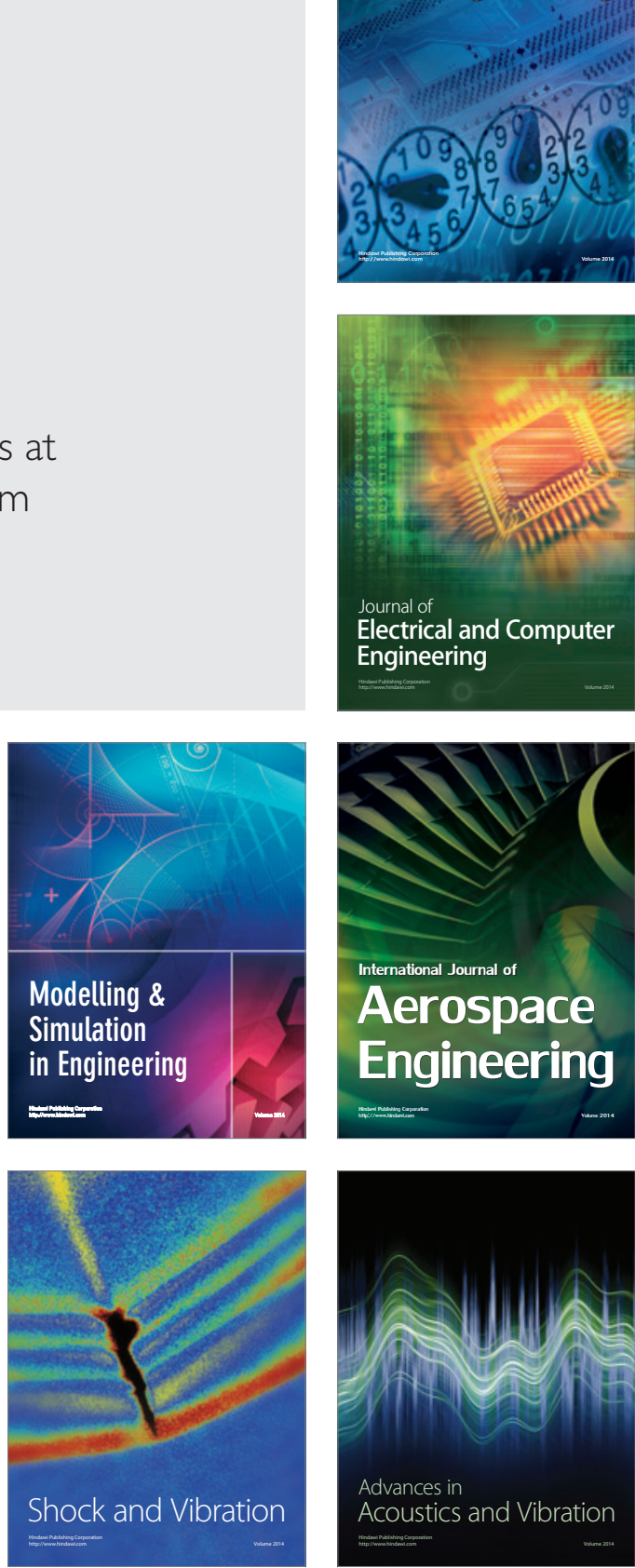